\title{
Comparison of saturation current characteristics for ultrathin silicon oxides grown on $n$ - and $p$-type silicon substrates simultaneously
}

\author{
Tsung-Miau Wang, Chia-Hua Chang, Shu-Jau Chang, and Jenn-Gwo Hwu ${ }^{\text {a) }}$ \\ Department of Electrical Engineering, National Taiwan University, Taipei, Taiwan, Republic of China \\ and Graduate Institute of Electronics Engineering, National Taiwan University, Taipei, Taiwan, \\ Republic of China
}

(Received 19 August 2005; accepted 2 August 2006; published 10 October 2006)

\begin{abstract}
Rapid thermal oxidations were simultaneously performed on $n$ - and $p$-type silicon substrates to investigate the saturation currents of metal-oxide-semiconductor (MOS) capacitors. For MOS capacitors on $n$-type $\mathrm{Si}$ substrates, the curves of capacitance versus gate voltage $(C-V)$ show almost no fixed charge, no lateral nonuniformity, and little interface trap density $\left(D_{\mathrm{it}}\right)$. The mechanism of the generation of the saturation current is recombination, and was investigated by electroluminescence. Also, the saturation current decreases as the oxide becomes thicker. However, the oxidation temperature must be sufficiently high to form high-quality oxide on $p$-type $\mathrm{Si}$ substrate. Controlled by minority carrier generation, the saturation current of the MOS $(p)$ capacitor also depends on $D_{\text {it }}$, suboxide, and bulk trap density. The saturation current increases with the thickness of the oxide. The generation mechanism of the saturation currents of MOS $(p)$ capacitors was also investigated by observing their dependencies on temperature. The mechanisms of the generation saturation currents of MOS capacitors grown on $n$ - and $p$-type Si substrates are basically different. () 2006 American Vacuum Society. [DOI: 10.1116/1.2345648]
\end{abstract}

\section{INTRODUCTION}

The miniaturization of electronic devices has led to the necessity for ultrathin silicon dioxide $\left(\mathrm{SiO}_{2}\right)$ layers with a thickness below $3 \mathrm{~nm}^{1}$ Among recent technological challenges is the maintenance of high-quality ultrathin dielectric layers. ${ }^{2,3}$ Silicon oxidation is currently one of the problematic areas in semiconductor science and technology. ${ }^{4,5}$ Growing thermal oxide grown in dry oxygen $\left(\mathrm{O}_{2}\right)$ is generally believed to be the most effective means of forming a highquality gate dielectric film, especially for mass production in industry. ${ }^{6,7}$ However, the intrinsic properties of ultrathin oxides grown on $n$ - and $p$-type substrates have seldom been addressed.

This work investigates the electrical characteristics of metal-oxide-semiconductor (MOS) capacitors with ultrathin oxides grown on $n$ - and $p$-type Si substrates, as in MOS $(n)$ and $\operatorname{MOS}(p)$ capacitors. The currents in deep depletion regions of both $\operatorname{MOS}(n)$ and $\operatorname{MOS}(p)$ capacitors are saturated. ${ }^{8,9}$ Notably, the current versus gate voltage $(I-V)$ curves of MOS $(n)$ and MOS $(p)$ capacitors, and especially the saturation currents, differ greatly. For MOS $(n)$ capacitors, the saturation current is attributed mainly to the electron-hole pair recombination mechanism. ${ }^{10}$ It can be examined by the electroluminescent (EL) method. ${ }^{11-14}$ However, for MOS $(p)$ capacitors, the saturation current is attributed mainly to the electron-hole pair generation mechanism, and is also controlled by $D_{\mathrm{it}}$, bulk traps, and the suboxide. ${ }^{15-18}$ As the oxide becomes thicker, the saturation current increases because the oxide becomes thicker. ${ }^{19-22}$ The generation mechanism can be confirmed by observing

a) Author to whom correspondence should be addressed; electronic mail: hwu@cc.ee.ntu.edu.tw the temperature dependence of saturation currents. Increasing the oxidation temperature improves the oxide quality of the MOS $(p)$, the result of which is confirmed by the curves of capacitance versus gate voltage $(C-V)$. This observation is interesting and useful in the study of ultrathin oxides.

\section{EXPERIMENT}

After standard Radio Corporation of America cleaning and HF dipping were performed, $n$-type and $p$-type (100) silicon wafers with a resistivity of $1-5 \Omega \mathrm{cm}$ were simultaneously oxidized in a rapid thermal processor at 900, 950, and $1000{ }^{\circ} \mathrm{C}$ for $30 \mathrm{~s}$ at 40 Torr. This process ensured that the two wafers had the same oxidation conditions. Thereafter, aluminum was evaporated onto each wafer and the metal gates were patterned by conventional photolithography. The areas of the devices were $150 \times 150 \mu \mathrm{m}^{2}$. The back side of each wafer was coated with an aluminum film to form the MOS capacitors after the back side oxides had been removed. The $n$-Si and $p$-Si wafers were denoted as chips 1 and 4, 2 and 5, and 3 and 6, respectively, at 900, 950 and $1000{ }^{\circ} \mathrm{C}$. Table I presents in detail the thicknesses of the oxides, which were determined by the quantum-mechanical fitting of $C$ - $V$ curves, ${ }^{23,24}$ except for chip 4, because the $\operatorname{MOS}(p)$ capacitors on chip 4 were too leaky to be fitted by a theoretical $C$ - $V$ quantum-mechanical (QM) curve. The oxide thickness of chip 4 is estimated to be $1.8 \mathrm{~nm}$ by current magnitude fitting at the accumulation region, based on the literature. $^{25}$

\section{RESULTS AND DISCUSSION}

In Fig. 1, the $C-V$ characteristic of MOS $(n)$ on chip 1 shows almost ideal behavior by comparison with the $C-V$ 
TABLE I. Oxidation conditions and oxide thicknesses for $n$ - and $p$-type wafers.

\begin{tabular}{ccc}
\hline \hline $900{ }^{\circ} \mathrm{C}$ & $n$ type & $1.9 \mathrm{~nm}$; chip 1 \\
$30 \mathrm{~s}$ & $p$ type & $1.8 \mathrm{~nm}$; chip 4 \\
$40 \mathrm{Torr}$ & $n$ type & $2.2 \mathrm{~nm}$; chip 2 \\
$950{ }^{\circ} \mathrm{C}$ & $p$ type & $2.1 \mathrm{~nm}$; chip 5 \\
$30 \mathrm{~s}$ & $n$ type & $2.3 \mathrm{~nm}$; chip 3 \\
$40 \mathrm{Torr}$ & $p$ type & $2.4 \mathrm{~nm}$; chip 6 \\
$1000{ }^{\circ} \mathrm{C}$ & \\
$30 \mathrm{~s}$ & \\
$40 \mathrm{Torr}$ &
\end{tabular}

characteristic of the MOS ( $p$ ) capacitor on chip 4. The $C$-V characteristic of the MOS $(p)$ capacitor on chip 4 exhibits serious flatband shift, lateral nonuniformity, and frequency dispersion among $1 \mathrm{MHz}, 100 \mathrm{kHz}$, and $10 \mathrm{kHz}$, as revealed by comparison with the theoretical QM curve. The capacitance falls in the accumulation bias region when the measurement frequency is $1 \mathrm{MHz}$, because the oxide is too leaky. In contrast, the capacitance increases dramatically in the accumulation region when the frequency is 100 and $10 \mathrm{kHz}$, because the series-resistance effect occurs. Around the accumulation region $\left(V_{g}=-1 \mathrm{~V}\right)$ is serious lateral nonuniformity. Many fixed charges are believed to distribute nonuniformly in the $\mathrm{SiO}_{2}$ bulk region. The capacitor can be viewed as numerous small subcapacitors connected in parallel. However, each small capacitor has its own fixed charges, stretching out the $C-V$ curve. The flatband shift $\left(-1 \mathrm{~V}<V_{g}\right.$ $<-0.4 \mathrm{~V})$ also indicates that the quality of the $\mathrm{Si} / \mathrm{SiO}_{2}$ interface of the MOS $(p)$ capacitor on chip 4 is poor.

If the oxidation temperature increases to $950{ }^{\circ} \mathrm{C}$, the $C$ - $V$ characteristics will be much improved, especially for the MOS $(p)$ capacitor. In Fig. 2, the $C-V$ characteristic of the $\operatorname{MOS}(n)$ capacitor on chip 2 exhibits no flatband voltage shift and less frequency dispersion between $1 \mathrm{MHz}$ and $10 \mathrm{kHz}$. The performance of the device on chip 2 is similar to that on chip 1. However, the $C-V$ curve of the MOS $(p)$ capacitor on chip 5 is much better than that on chip 4 . Near

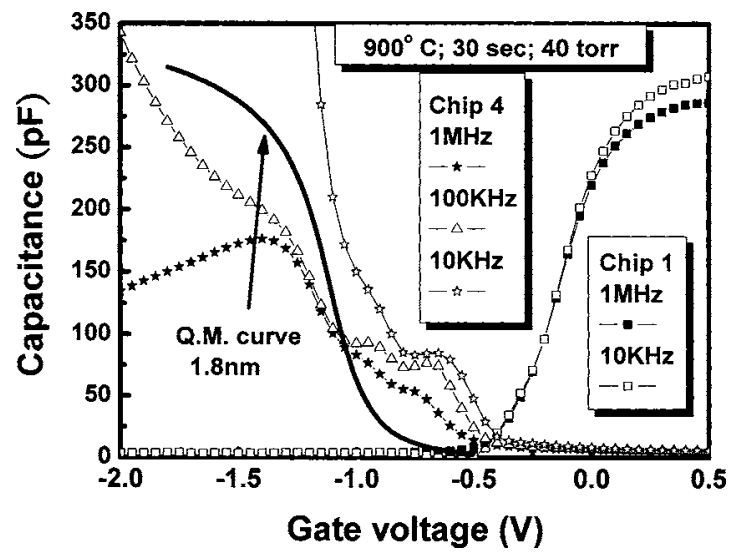

FIG. 1. $1 \mathrm{MHz}$ and $10 \mathrm{kHz} C-V$ characteristics of the MOS capacitors on chips 1 and 4. Also, the $100 \mathrm{kHz} C-V$ characteristics of the MOS ( $p$ ) capacitors on chip 4 and the theoretical QM curve are attached.

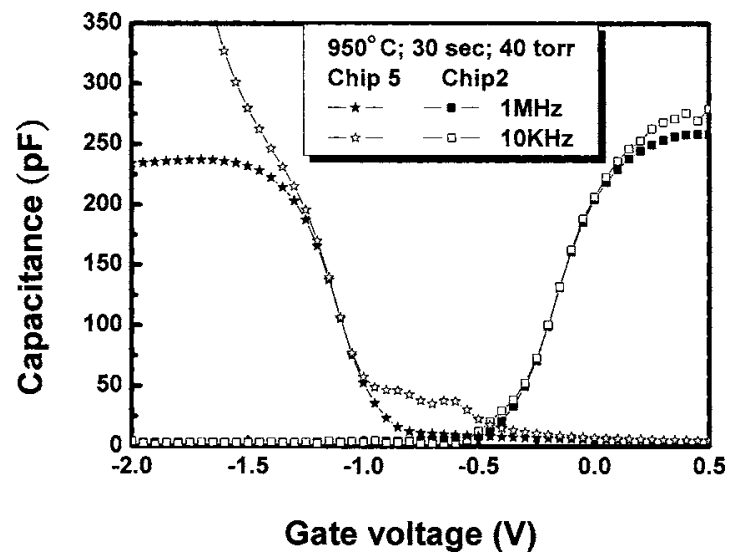

FIG. 2. $1 \mathrm{MHz}$ and $10 \mathrm{kHz} C-V$ characteristics of the MOS capacitors on chips 2 and 5 .

the flatband voltage, the amount of $D_{\mathrm{it}}$ is greatly suppressed. The lateral nonuniformity also disappears. As the oxidation temperature becomes higher, the above observation is again verified at an even higher oxidation temperature of $1000{ }^{\circ} \mathrm{C}$. In Fig. 3, chips 3 and 6 have oxide of a higher quality than that in the chips in Figs. 1 and 2. However, the thicker oxide $(2.4 \mathrm{~nm})$ and lower frequency $(10 \mathrm{kHz})$ cause the interface states $\left(D_{\text {it }}\right)$ to respond to the frequency and contribute to the increase in the measured capacitance in the beginning of the deep depletion region. As the frequency increases, the $D_{\text {it }}$ effect decreases.

The currents of MOS capacitors with ultrathin oxides are saturated in the deep depletion region. ${ }^{9,14-20}$ The corresponding $C$ - $V$ curves exhibit deep depletion behavior, as demonstrated in Figs. 1 and 2. Deep depletion is caused by the lack of minority carriers, and increases the width of the depletion region. The occurrence of a deep depletion is revealed by the decreasing capacitance in the $C-V$ curves. All of the $C-V$ curves in Figs. 1 and 2 exhibit behaviors. Here, the difference between "inversion" and "saturation" must be emphasized. Inversion refers to the accumulation of a mass of electrons near the $\mathrm{SiO}_{2} / \mathrm{Si}$ interface when the oxide is thick. However, if the oxide is thinner than $3 \mathrm{~nm}$, then it is too

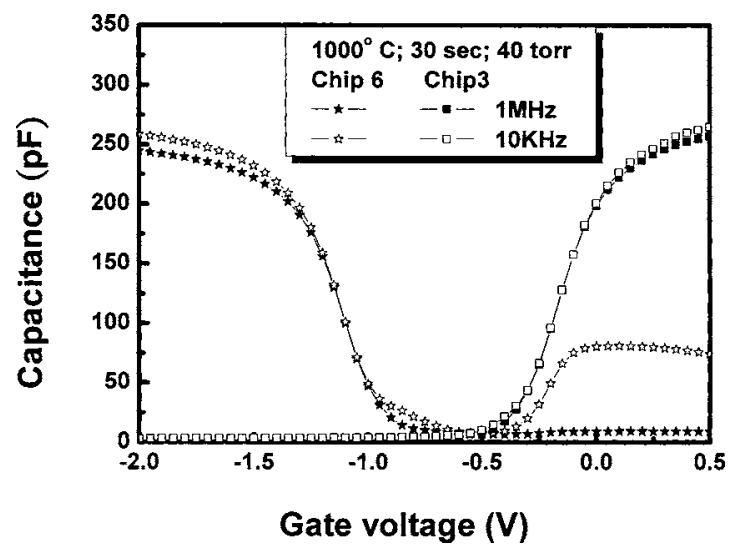

FIG. 3. $1 \mathrm{MHz}$ and $10 \mathrm{kHz} C-V$ characteristics of the MOS capacitors on chips 3 and 6 . 
Recombination Mechanism

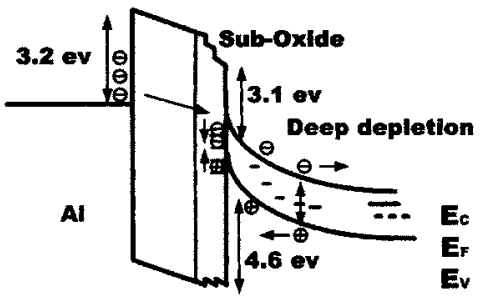

Metal OxIde N-SI Sub

(a)

Generation Mechanism

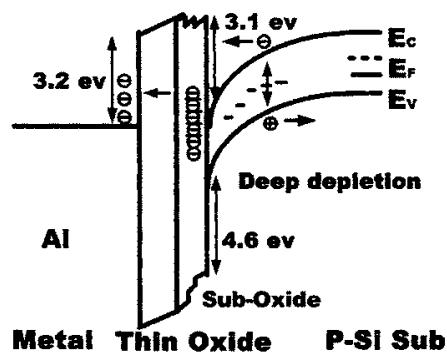

(b)

FIG. 4. Current mechanisms of MOS capacitors on (a) $n$-type substrate and (b) $p$-type substrate when biased under deep depletion region.

leaky and electrons tunnel through it directly. The minority carriers cannot be maintained at thermal equilibrium, causing deep depletion. Figure 4(a) shows the energy band diagram of MOS $(n)$ in the current saturation region (or deep depletion region). When the electrons tunnel through the oxide, some are trapped in the suboxide region or the interface region. Meanwhile, electron-hole pairs are generated in the deep depletion region in $n$-type $\mathrm{Si}$ substrate. The generated electrons drift to the neutral region in the $n$-type $\mathrm{Si}$ substrate and the holes drift to the $\mathrm{Si} / \mathrm{SiO}_{2}$ interface. The electrons from the metal gate and the minority hole carriers from the deep depletion region quickly recombine in either the suboxide region or the $\mathrm{Si} / \mathrm{SiO}_{2}$ interface region. Recombination occurs in the Si substrate near the interfacial region. However, for the $p$-Si substrate, the saturation current mechanism is different. Figure 4(b) shows the conducting mechanism of the MOS $(p)$ capacitors in the deep depletion region. Initially, the electron-hole pairs are generated by thermal or photonic excitation in the deep depletion region of the $\mathrm{Si}$ substrate. The generated electrons drift to the $\mathrm{Si} / \mathrm{SiO}_{2}$ interface and holes drift to the neutral region in the $p$-type $\mathrm{Si}$ substrate. The electrons are trapped in the suboxide region and the interface region. If the electrons have sufficient energy, such as at high temperature or when accelerated by an electrical field, they are detrapped and tunnel through the oxide. The current is limited by the generation of minority electron carriers. Also, the traps near the $\mathrm{Si} / \mathrm{SiO}_{2}$ contribute to the saturation current. In this case, the current increases with the number of traps in the suboxide and $D_{\mathrm{it}}$. If the oxide

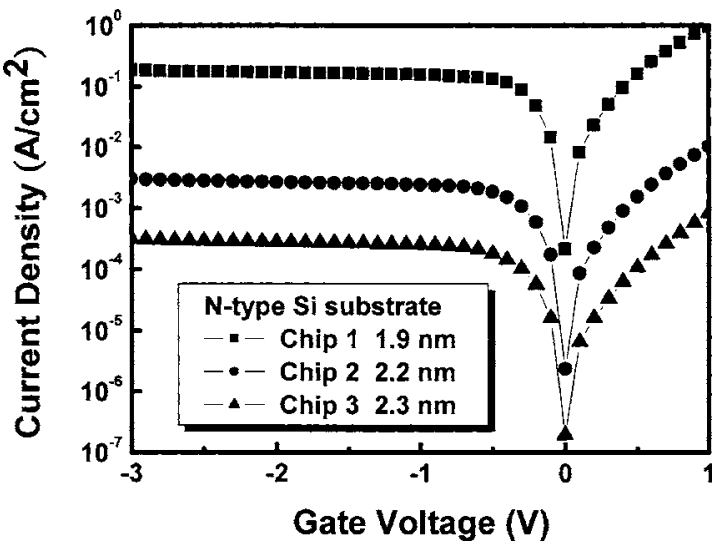

FIG. 5. Typical $I-V$ characteristics of the MOS $(n)$ capacitors on chips 1-3.

becomes thicker, then the suboxide region also becomes thicker, increasing the current density in the saturation region in $\operatorname{MOS}(p)$ capacitors. ${ }^{21}$

Figure 5 plots the typical $I-V$ characteristics of MOS $(n)$ capacitors with oxides of various thicknesses. As the oxide thickness increases, the current magnitude decreases. The current magnitude is limited by the tunneling rate, which is determined by the oxide thickness. A thicker oxide yields a smaller current. In contrast, Fig. 6 plots the $I-V$ curves of the $\operatorname{MOS}(p)$ capacitor. Chips 4,5 , and 6 have oxide thicknesses of $1.8,2.1$, and $2.4 \mathrm{~nm}$, respectively. The saturation current increases with the thickness of the oxide, because the suboxide thickness increases with the total oxide thickness. Therefore, when the oxide becomes thicker, the suboxide region also becomes thicker, increasing the number of traps, thereby increasing the leakage current. ${ }^{21}$

Finally, optical and electrical analyses are performed to verify the above current mechanisms. Here, the EL technique was applied as a powerful tool to investigate the electronhole recombination mechanism, especially for $\operatorname{MOS}(n)$ capacitors. The plots of current versus temperature are used to identify the electron-hole pair generation process, especially for MOS ( $p$ ) capacitors. Figure 7(a) plots the EL intensity of the MOS $(n)$ capacitor under a saturation current bias. The normalized intensity spectrum exhibits a clear peak, with an

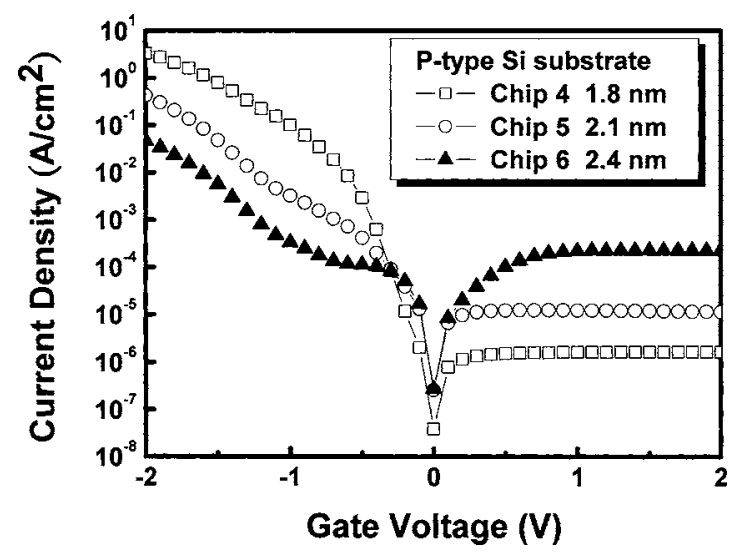

FIG. 6. Typical $I-V$ characteristics of the $\operatorname{MOS}(p)$ capacitors on chips 4-6. 


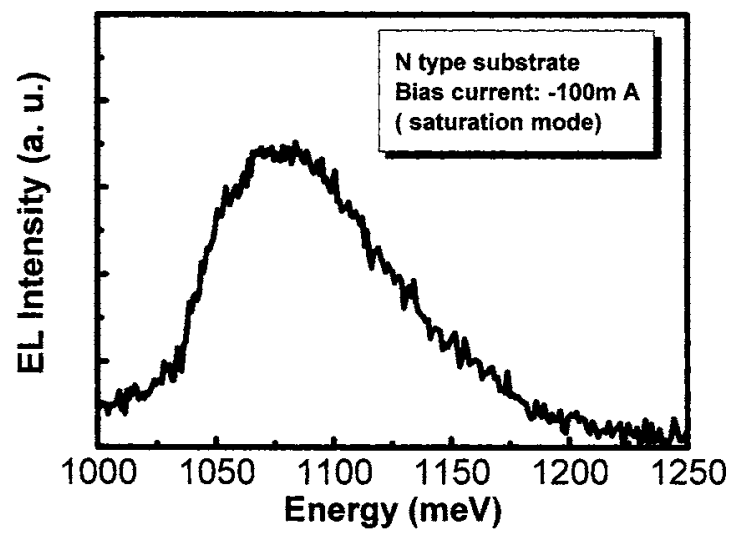

(a)

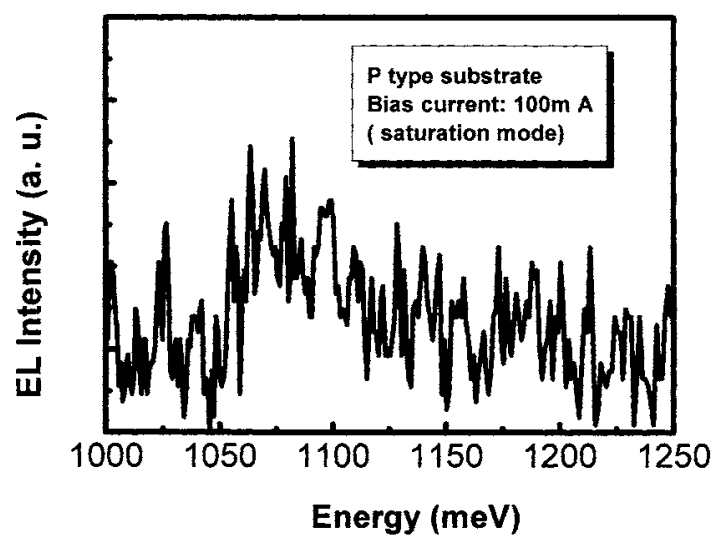

(b)

FIG. 7. EL intensity distributions of (a) MOS ( $n$ ) capacitor and (b) MOS ( $p$ ) capacitor.

energy of approximately $1.1 \mathrm{eV}$, which is close to the band gap of the Si substrate. Hence, radiative recombination occurs. Electrons in the conduction band drop to the valence band and recombine with the holes. The process occurs in the deep depletion region. The recombination process is supposed to be the main current mechanism of MOS $(n)$ capacitors under saturation bias. The recombination process is both radiative and nonradiative. The radiative process can be measured by the EL method and the emission of photons. However, the nonradiative process occurs as the electron-hole pairs recombine via the $\mathrm{Si}$ bulk traps, via $D_{\mathrm{it}}$, and in the suboxide region. However, the nonradiative process emits phonons. Figure 7(b) shows the EL intensity for the MOS ( $p)$ capacitor under current saturation. Clearly, no EL intensity is observed, indicating an absence of recombination as the main current mechanism for $\operatorname{MOS}(p)$ capacitors in the saturation region.

The current generation mechanisms were investigated by varying the temperature. Figure 8 plots the saturation current density at a certain bias versus oxide thickness at various temperatures for chips 1-6. Previous works have demonstrated that increasing the temperature accelerates generation of electron-hole pair. $\operatorname{MOS}(n)$ and $\operatorname{MOS}(p)$ capacitors exhibited different dependencies on temperature. ${ }^{15}$ The temperatures ranged from 30 to $80^{\circ} \mathrm{C}$. For the MOS $(p)$ capaci-

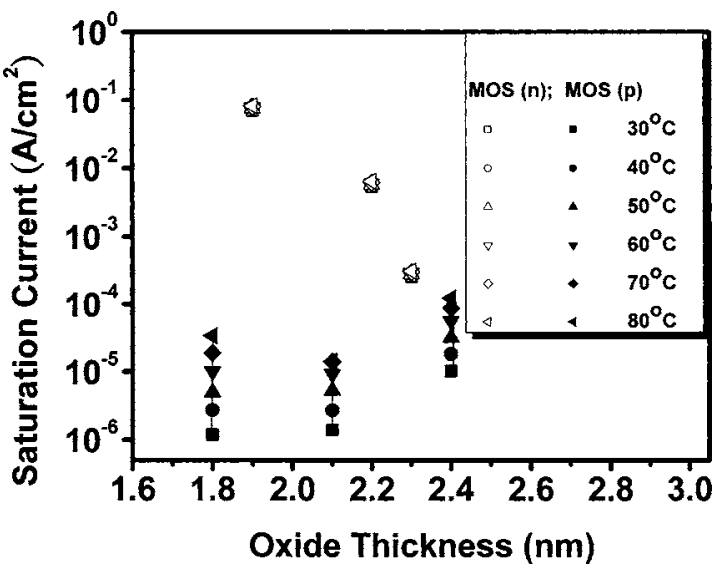

FIG. 8. Saturation current density vs oxide thicknesses under various temperatures of $\operatorname{MOS}(n)$ and $\operatorname{MOS}(p)$ capacitors biased at $V_{G}=V_{\mathrm{FB}}-2 \mathrm{~V}$ and $V_{G}=V_{\mathrm{FB}}+2 \mathrm{~V}$, respectively.

tors, the saturation currents are very sensitive to temperature. For $\operatorname{MOS}(n)$ capacitors, the saturation currents are almost independent of temperature. Different current mechanisms, including generation and recombination processes, are responsible for the behaviors of $\operatorname{MOS}(p)$ and $\operatorname{MOS}(n)$ capacitors, respectively. This fact is of importance in studies of ultrathin oxides.

\section{CONCLUSION}

In this investigation, in the very early stages of oxidation, $n$-type $\mathrm{Si}$ was oxidized more completely than $p$-Si at low temperature. The $C-V$ curves of $\operatorname{MOS}(n)$ capacitors show less flatband voltage shift, fewer fixed charges, and less lateral nonuniformity. However, for $p$-type $\mathrm{Si}$, if the oxidation temperature is not sufficiently high, then the quality of the oxide will be poorer than that of the $n$ type. Furthermore, for $\operatorname{MOS}(n)$ capacitors in the saturation current region, the current is dominated by electron-hole recombination and can be examined by the EL method. However, the generation of the minority electron carriers is the main factor in determining the saturation current of the MOS $(p)$ capacitors. The current magnitude increases with the thickness of the oxide. The electron-hole pair generation mechanism was examined by observing the temperature dependencies of the saturation currents. $n$ - and $p$-type Si substrates provide different saturation current mechanisms for MOS capacitors with ultrathin oxides.

\section{ACKNOWLEDGMENT}

The authors would like to thank the National Science Council of the Republic of China, Taiwan, for financially supporting this research under Contract No. NSC94-2215-E002-004.

${ }^{1}$ C.-H. Lin, B.-C. Hsu, M. H. Lee, and C. W. Liu, IEEE Trans. Electron Devices 48, 2125 (2001)

${ }^{2}$ E. M. Vogel, M. D. Edelstein, and J. S. Suehle, J. Appl. Phys. 90, 2338 (2001).

${ }^{3}$ W. Mizubayashi and S. Miyazaki, Jpn. J. Appl. Phys., Part 1 43, 6925 
(2004).

${ }^{4}$ M. Uematsu, H. Kageshima, and K. Shiraishi, Comput. Mater. Sci. 24, 229 (2002).

${ }^{5}$ A. Vedda, M. Martini, and G. Spinolo, J. Appl. Phys. 90, 5013 (2001).

${ }^{6}$ R. Deaton and H. Z. Massoud, IEEE Trans. Semicond. Manuf. 5, 347 (1992).

${ }^{7}$ Y. Kotani and Y. Omura, Jpn. J. Appl. Phys., Part 1 39, 4549 (2000).

${ }^{8}$ W. C. Lee and Chenming Hu, Tech. Dig. VLSI Symp. 2000, 198.

${ }^{9}$ M. Y. Doghish and F. D. Ho, IEEE Trans. Electron Devices 40, 1446 (1993).

${ }^{10}$ S. J. Robinson, S. R. Wenham, P. P. Altermatt, A. G. Aberle, and G. Heiser, J. Appl. Phys. 78, 4740 (1995).

${ }^{11}$ V. R. Nikitenko and J. M. Lupton, J. Appl. Phys. 93, 5973 (2003).

${ }^{12}$ J. Kalinowski, L. C. Picciolo, H. Murata, and Z. H. Kafafi, J. Appl. Phys. 89, 1866 (2001).

${ }^{13}$ D. V. Khramtchenkov, V. I. Arkhipov, and H. Bässler, J. Appl. Phys. 81, 6954 (1997)

${ }^{14}$ Y.-H. Tak and H. Bässler, J. Appl. Phys. 81, 6963 (1997).
${ }^{15}$ Y.-H. Shih, S.-R. Lin, T. M. Wang, and J.-G. Hwu, IEEE Trans. Electron Devices 51, 1514 (2004).

${ }^{16}$ C.-C. Hong and J.-G. Hwu, IEEE Electron Device Lett. 24, 408 (2003).

${ }^{17}$ C.-C. Hong, W.-J. Liao, and J.-G. Hwu, Appl. Phys. Lett. 82, 3916 (2003).

${ }^{18}$ C.-C. Hong, C.-Y. Chang, C.-Y. Lee, and J.-G. Hwu, J. Appl. Phys. 93, 2225 (2003).

${ }^{19}$ T. M. Wang and J.-G. Hwu, J. Appl. Phys. 97, 44504 (2005).

${ }^{20}$ K. J. Yang and C. Hu, IEEE Trans. Electron Devices 46, 1500 (1999).

${ }^{21}$ Y.-P. Lin and J.-G. Hwu, J. Vac. Sci. Technol. 22, 2256 (2004).

${ }^{22}$ C.-C. Hong, J.-L. Chen, and J.-G. Hwu, J. Vac. Sci. Technol. A 20, 2 (2002).

${ }^{23}$ N. Yang, W. K. Henson, and J. R. Hauser, IEEE Trans. Electron Devices 46, 1464 (1999).

${ }^{24}$ K. J. Yang and C. Hu, IEEE Trans. Electron Devices 46, 1500 (1999).

${ }^{25}$ K. Sekine, S. Inumiya, M. Sato, A. Kaneko, K. Eguchi, and Y. Tsunashima, Tech. Dig. - Int. Electron Devices Meet. 2003, 102. 